\title{
Non-DESTRUCTIVE DNA EXTRACTION FROM ChIRONOMIDAE, INCLUdiNG OF FRAGILE PUPAL EXUVIAE, EXTENDS ANALYSABLE COLLECTIONS AND ENHANCES VOUCHERING
}

\author{
Matthew N. Krosch ${ }^{1}$, Peter S. Cranston ${ }^{2}$ \\ ${ }^{1}$ School of Earth, Environmental and Biological Sciences, Queensland University of Technology, \\ Brisbane, 4001, Australia \\ E-mail:kroschie@gmail.com \\ ${ }^{2}$ Evolution, Ecology and Genetics, Research School of Biology, The Australian National University, \\ Canberra, 0200, Australia
}

\section{Introduction}

It is well established that the traditional taxonomy and nomenclature of Chironomidae relies on adult males whose usually characteristic genitalia provide evidence of species distinction. In the early days some names were based on female adults of variable distinctiveness - but females are difficult to identify (Ekrem et al. 2010) and many of these names remain dubious. In Russia especially, a system based on larval morphology grew in parallel to the conventional adult-based system. The systems became reconciled with the studies that underlay the production of the Holarctic generic keys to Chironomidae, commencing notably with the larval volume (Wiederholm, 1983). Ever since Thienemann's pioneering studies, it has been evident that the pupa, notably the cast skins (exuviae) provide a wealth of features that can aid in identification (e.g. Wiederholm, 1986). Furthermore, the pupae can be readily associated with name-bearing adults when a pharate ('cloaked') adult stage is visible within the pupa. Association of larvae with the name-bearing later stages has been much more difficult, time-consuming and fraught with risk of failure. Yet it is identification of the larval stage that is needed by most applied researchers due to the value of the immature stages of the family in aquatic monitoring for water quality, although the pupal stage also has advocates (reviewed by Sinclair \& Gresens, 2008). Few use the adult stage for such purposes as their provenance and association with the water body can be verified only by emergence trapping, and sampling of adults lies outside regular aquatic monitoring protocols.

An answer to this mismatch in demand and taxonomic expertise may lie with the actual and potential use of molecular data. In the past two decades researchers have used data derived from DNA to estimate phylogenies for their inherent interest (e.g. Guryev et al. 2001), in supplementing morphological characters (Cranston et al. 2012), in estimation of support for biogeographic hypotheses (e.g. Krosch et al. 2009; Cranston et al. 2010) and increasingly to propose identification of any or all stages (Carew et al. 2007; Ekrem et al. 2007). In the realm of understanding midge diversity and community structure, cryptic diversity has been detected (Carew et al. 2005) including by use specifically of a 'DNA barcoding' approach (Pfenninger 2007; Sinclair \& Gresens 2008; Ekrem et al. 2010). Phylogeographic molecular genetic studies have been undertaken to reveal the origin and maintenance of chironomid populations (e.g. Martin et al. 2002; Krosch et al. 2009; Kaiser et al. 2010) and fine-scale patterns of gene flow among adjacent streams have been assessed (Krosch et al. 2011a). Early on, adult stages were used in such studies with immature stages incorporated in some. Hence, many techniques for DNA extraction from single chironomid specimens were developed specifically for adult bodies and, thus, relatively larger amounts of tissue (e.g. Willassen 1999; Wang \& Wang 2012). Although molecular techniques have been used to associate the sexes of adults (Willassen 2005), explicit attempts made to associate previously unknown immature stages with adult stages have been few. Species associations were either 'known' from cultivation (e.g. Chironomus, Guryev et al. 2001) or by rearing in the field (e.g. Archaeochlus / Austrochlus, Cranston et al. 2002) or in the laboratory (Sinclair \& Gresens 2008). Targetted 'taxonomic' associations of life histories solely via DNA have been often incidental and remain rare in chironomid studies.

In seeking to maximize numbers and/or diversity of taxa for large-scale molecular phylogenetic and population genetics studies, we sought the aquatic immature stages, using conventional techniques with aquatic nets and kicking substrates or interception of drifting individuals (Cranston et al. 2010, 2012; Krosch et al. 2011a, b). In over 2 decades of working intermittently with molecular aspects of Chironomidae, between us we believe we have encountered most problems, solved many, and have unpublished insights into what can go wrong, what works well, and what can be im- 
proved in our protocols. Largely in response to requests from interested colleagues, we describe here the simple technique that makes possible retrieval of PCR-quality DNA from chironomids including even from single pupal exuviae. Previous attempts to extract DNA from exuviae using a modified salting out protocol (Miller et al. 1988) failed, and we assumed such specimens lacked sufficient remaining tissue post-adult emergence for DNA extraction. However with careful handling of specimens and more sensitive modern extraction techniques described below, good DNA can be recovered, thereby allowing access to greater numbers of immature stages, and avoiding the need for laborious and often unrewarding rearing, making molecular associations between life stages simpler.

\section{Methods}

Field

We use all traditional and some less usual sampling when collecting material in the field, according to the purpose. For population genetics studies we sample intensively in a narrowly defined area containing the appropriate microhabitat(s), seeking known target taxa. For phylogenetic studies in which we seek also to maximise diversity, we sample all visible microhabitats. At all lotic sites we place a drift net downstream of other collecting activity for the duration of the visit, or longer, including overnight if feasible, for pupal including exuvial sampling. In contrast to most studies, we sort specimens from the substrate at or close to the sampling location using dissector microscopes. Live sorting is preferable with specimens being picked with fine forceps and placed immediately into isopropanol or molecular grade ethanol, and never into formalin, methanol or ethanol that has in any way been 'denatured'. Only if a sample cannot be sorted 'live' in timely manner do we preserve in alcohol, after removal of larger particulate matter and sieving for medium-sized organic removal. Isopropanol, although more expensive, is widely available for use in molecular labs, preserves DNA very well, is less volatile and less flammable (important if flights are involved in field work) and is not hygroscopic - your $100 \%$ alcohol remains 'absolute'. Most failures to extract DNA from specimens are associated with the age and storage of older material (which should be kept cold and dark) or unwitting use of certain denatured ethanols as supplied to many 'wet labs'. We suspect that some total DNA destruction is caused by customs irradiation of international mailed material but cannot verify this.

For some taxa, life history stage and location it is possible to know the specimen identity prior to laboratory processing for molecular work. For instance, reared monoculture such as species of Chironomus may be assumed to be that species, and those familiar with the fauna of a particular area can identify much without slide preparation. However as we know, accurate identification using morphology requires slide preparation, which is prima facie incompatible with DNA extraction. For larvae we amputate the head and prepare it as a temporary slide mount (in Hoyers mountant) for rapid identification prior to decision on DNA extraction: the same applies to male genitalia. If sequenced, these vouchers are remounted in Euparal for permanence. For pupae, identification often can be made without prior slide preparation.

\section{Laboratory}

We extract total genomic DNA from specimens using a Qiagen DNeasy® Blood and Tissue Kit (Qiagen, Hilden, Germany) following manufacturer's protocol. Modifications to this protocol were as follows: (1) tissue was digested with proteinase $\mathrm{K}$ overnight at $37^{\circ} \mathrm{C}$; (2) after digestion, cuticles were removed carefully using fine-tipped forceps and transferred to $90 \%$ isopropanol before vouchering as slides in Euparal; (3) the final elution volume was $100 \mu \mathrm{L}$ to increase the final DNA concentration of the eluate; and (4) the incubation time for elution was increased to 5 minutes. Furthermore, digestions were never vortexed while cuticles remained in solution to avoid damage to diagnostic morphological characters. At all stages, care with handling is essential and we found using the solution's surface tension, rather than direct pressure from forceps, to be sufficient for transferring specimens between micro-centrifuge tubes. We anticipate that extraction kits from other manufacturers may produce similar results under the same modification, but this remains untested currently.

We report extraction success from pupal exuviae only here and do so simply in terms of reporting successful amplification and sequencing of the barcode region of the mitochondrial COI gene and the slower evolving nuclear $28 S$ rRNA region. We acknowledge that using more stringent quality control measures (e.g. NanoDrop or BioAnalyzer technologies) may provide more information about actual DNA concentration and quality. However, we envisage that the majority of end-users of extracted DNA from pupal exuviae will be interested in utilising DNA for traditional PCR and Sanger sequencing and thus we believe our qualitative assessment valid in this case. Reactions were carried out in a total volume of $25 \mu \mathrm{L}$, containing $2-5 \mu \mathrm{L}$ 


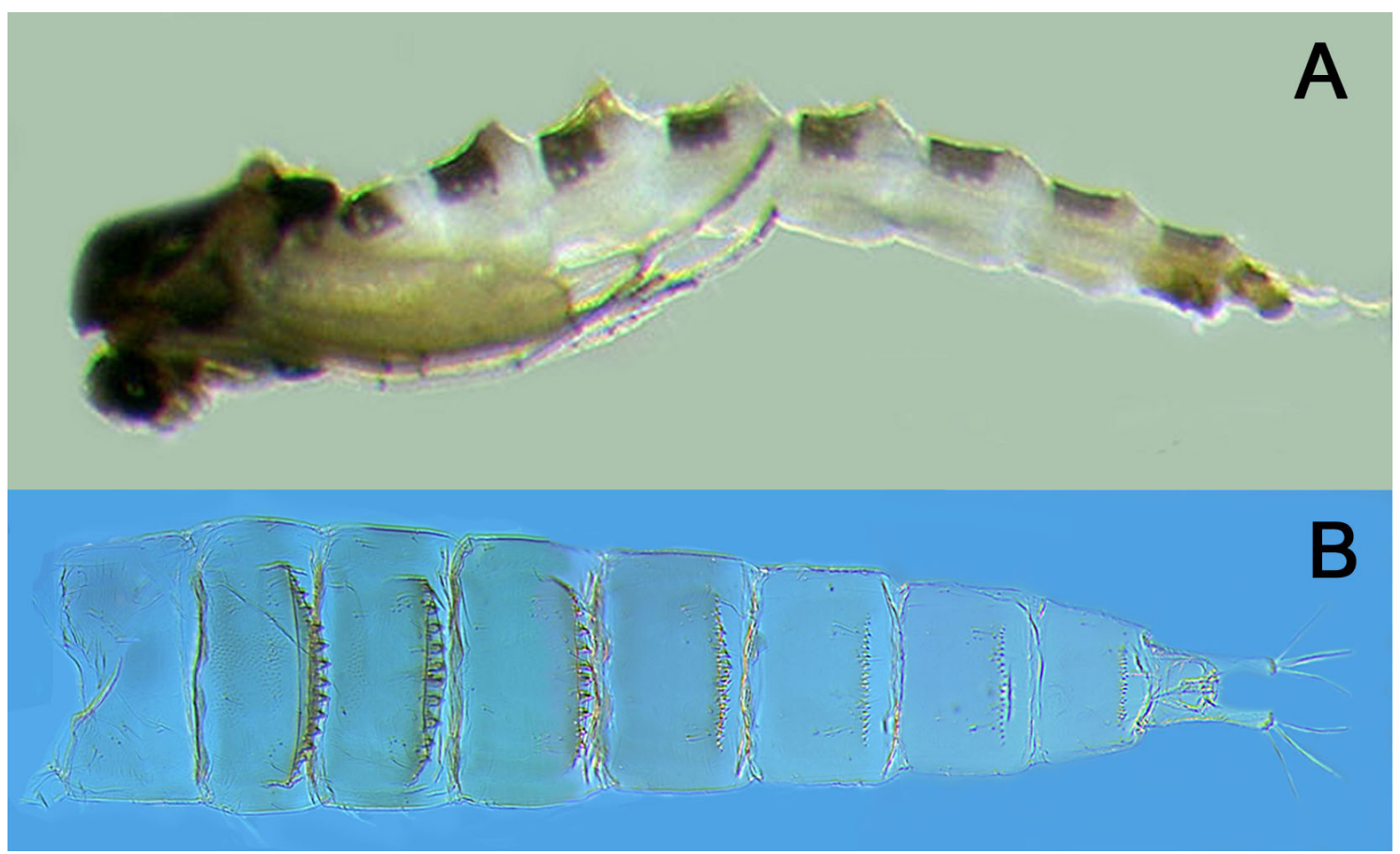

A. Pharate adult pupa of Stictocladius lacuniferus Freeman, from New Zealand. Note the leg sheaths are all extended and none recurved under wing sheath. B. Pupal exuviae: abdomen of Stictocladius uniserialis Freeman, from Australia. The genus Stictocladius, following morphological review by Cranston \& Sæther (2010) and Sæther \& Cranston (2012) is now the subject of molecular study by Matt Krosch and Peter Cranston. The pupal stage not only provides good morphological features, but has provided valuable DNA even from exuviae, as outlined in the article.

of template DNA, $0.6 \mu \mathrm{L}$ of each primer $(10 \mathrm{pmol} /$ $\mu \mathrm{L}$ - manufactured by Geneworks, Adelaide, Australia), 1.75-3.5 $\mu \mathrm{L}$ of of $5 \mathrm{X}$ MyTaq Red Reaction Buffer (Bioline, London, UK) and $0.2 \mu \mathrm{L}$ of MyTaq DNA Polymerase (Bioline). Primer sequences and PCR protocols for both genes can be found in Krosch et al. (2011b). Amplified products were purified using an ISOLATE PCR and Gel Kit (Bioline) according to manufacturer's protocols, but using only $13 \mu \mathrm{L}$ of elution buffer and with a longer incubation time following addition of elution buffer to increase final concentration of amplified fragments. As with DNA extraction, choice of manufacturer of kits for the purification of amplified products is likely not to influence success rates greatly. Purified fragments were sequenced using ABI BigDye ${ }^{\circledR}$ Terminator v3.1 chemistry, precipitated using a standard ethanol-based protocol and analysed on an ABI3500 sequencing platform at the Molecular Genetics Research Facility (QUT, Brisbane). For more detailed information on methods and success of 'routine' DNA extraction from larvae or pupae we refer the reader to our existing publications cited below that use molecular data.

\section{Vouchering specimens}

In the early days, the few people working with chironomid DNA had a rather casual approach to vouchering material needed to verify that sequenc- es obtained actually derived from a named and retained specimen (Jon Martin U. of Melbourne, pers. comm, Peter Cranston, pers. obs.). This arose partially in the belief that DNA extraction from small insects involved grinding up (destroying) at least a complete specimen, sometimes more than one, because of the demands of the salting-out protocol (Miller et al. 1988). Maintenance of reliable vouchers followed with: 1. growing awareness that not all published sequences were what they were purported to be; 2. recognition that 'inessential' parts of a single specimen could provide enough DNA by newer extraction protocols, and the rest be retained as a voucher; 3 . recognition that the cuticle alone, after gentle extraction of DNA, provides a complete voucher; and 4. multiple specimens from natural populations should and need never be co-extracted. However, the latter point does remain viable for multiple specimens from laboratory culture lines or egg masses for use in downstream applications that require large quantities of DNA, such as optimisation of DNA systems or PCR primers.

Procedures are a little time-consuming and need accurate recording - we give each potential molecular specimen a unique 'site (year date) plus sequential numerical code' at the outset - whether or not DNA is obtained. This unique identifier is as- 
sociated with the DNA throughout processing, and with the cuticile obtained after extraction, or the slide-mounted larval head capsule if it is only the body that is taken through extraction. These slide mounted materials are treated with the same care in preparation, curation and specified repository as type material in a taxonomic study. The unique specifier code and associated data remains linked to the data submitted to Genbank (http://www. ncbi.nlm.nih.gov/genbank/).

\section{Results}

This technique, which already has worked well across other life stages from many subfamilies of Chironomidae, was trialed on exuvial specimens across a range of Orthocladiinae taxa. Of 58 exuvial specimens derived from separate field collections made at various sites across Australia during 2011 and 2012, extracted as described above, 27 have been successfully amplified and sequenced for $C O I$ and 17 for $28 S$. Sequence quality always was acceptable under standard QC metrics (e.g. signal strength) applied by the sequencing software and sequencing success was repeatable via both multiple attempts using the same purified PCR product and multiple initial PCR reactions. Moreover, vouchers made from cuticles post-digestion showed that diagnostic characters remained intact and morphology - and molecular-based identifications converged for all specimens. A number of specimens, in addition to those successfully sequenced for either locus, amplified successfully (as assessed using 1.5\% agarose check gels) but did not produce clean or useable sequence. This suggests that further optimisation of protocols may be necessary, especially with regard to the ratio of DNA template to primer in sequencing reactions.

It is not surprising that even this technique can fail to recover usable DNA from some specimens. Extraction of DNA from pupal exuviae relies on the presence of muscle tissue that is left behind on the inner surface of the cuticle by the emerging adult. DNA cannot be extracted from the cuticle itself. Thus, we suspect that the success of this technique is highly correlated with how long exuviae have been exposed to the aquatic environment after adult emergence. The longer the exposure time prior to collection, the greater the likelihood of any remaining muscle tissue being scoured or degraded. The same principle applies to the duration that specimens spend in alcohol collections. Furthermore, we note also problems encountered with degraded ethanol for preservation and quarantine of shipped specimens using X-rays (particularly to the USA). These factors act to shear DNA strands and greatly reduce efficiency of extraction and amplification, often to the point of complete failure for entire collections. Moreover, given the much higher copy number of mitochondrial genomes within each cell, we anticipated that DNA extracted from exuviae would perform better for amplifying mtDNA loci than nuclear. Although we did not observe a dramatic difference in total numbers sequenced for each locus here, $28 S$ amplification required more troubleshooting than $C O I$. The use of intermediate primers for COI, which often produce sequence data from older more degraded specimens (Jon Martin, pers. comm.) may allow even sub-optimal specimens to be used.

\section{Discussion}

The addition of PCR-quality DNA sequences from 'wild caught' pupal exuviae described here expands the number of sequenceable specimens for chironomid researchers who collect in aquatic environments. The greatest benefit of this technique will likely be in aiding the development of molecular associations between life stages of species by negating the need for rearing of larvae through to adults. This technique also has potential importance for those investigating population genetic structure, gene flow and dispersal where sample sizes must be maximised from each study site. We encourage other researchers to retain, and attempt to extract DNA from exuviae and not consider these important specimens valueless for molecular purposes.

However the addition of a 'new' life history stage, the pupal exuviae, to the repertoire of those engaged in molecular studies of Chironomidae should not obscure that even this suite of techniques do not provide the perfect answer to all questions. We note that there are sporadic and difficult to diagnose difficulties with extraction for some specimens or collection conditions in addition to the unknown age and history of collected exuviae, or interceptions of larvae, pupae and adults by netting. There are sporadic problems with amplification of DNA from mermithids rather than the midge host, apparently restricted to $18 \mathrm{~S}$ rRNA and probably due to primer degeneracy.

The procedures and protocols associated with specimen tracking, vouchering and truly geocoded and permanently labelled specimens are particularly important as we recognise increased cryptic diversity. Unique vouchers allow us to revisit actual specimens (and locations) of 'known' molecular identity and seek to validate perhaps obscure or overlooked morphology that will allow nonmolecular identification. Furthermore, GenBank 
is not prescriptive concerning vouchering and thus the database is error-ridden and requires validation and correction for many anomalous taxa. To date we have no complete and reliable library to test our species identifications (Ekrem et al. 2007) and thus morphological vouchers for DNA sequences remain as important as morphological types. Webb et al. (2012) illustrate a salutary case of difficulties in tracking down morphological vouchers for molecular databased N. American Ephemeroptera, despite the DNA barcode initiative's long-standing instructions concerning appropriate vouchering. DNA taxonomy does not over-ride the common sense understanding of how to go about systematic science, regarding repeatability and documentation of evidence.

\section{Acknowledgments}

Our molecular studies of Chironomidae have been funded mainly by an endowment by Evert Schlinger to the University of California at Davis (2000-2010). An NSF grant MIDGEPEET: A Collaborative Effort to Increase Taxonomic Expertise in Understudied Families of Nematocerous Diptera award 0933218 to PI John (Kevin) Moulton funded us to study Stictocladius, including to test these techniques on extraction from pupal exuviae. We thank Jon Martin for review, helpful comments and permission to cite from his experiences.

\section{References}

Carew, M.E., Pettigrove, V. and Hoffmann, A.A. 2005. The utility of DNA markers in classical taxonomy: using cytochrome oxidase I markers to differentiate Australian Cladopelma (Diptera: Chironomidae) midges. - Annals of the Entomological Society of America 98: 587594.

Carew, M.E., Pettigrove, V., Cox, R.L. and Hoffmann, A.A. 2007. DNA identification of urban Tanytarsini chironomids (Diptera: Chironomidae). - Journal of the North American Benthological Society 26: 587-600.

Cranston, P.S., Edward, D.H.D. and Cook, L.G. 2002. New status, distribution records and phylogeny for Australian mandibulate Chironomidae (Diptera). - Australian Journal of Entomology 41: 357-366.

Ekrem, T., Willassen, E. and Stur, E. 2007. A comprehensive DNA sequence library is essential for identification with DNA barcodes. - Molecular Phylogenetics and Evolution 43: 530-542.

Ekrem, T., Stur, E. and Hebert, P.D.N. 2010. Females do count: Documenting Chironomidae
(Diptera) species diversity using DNA barcoding. - Organismal Diversity and Evolution 10: 397-408.

Guryev, V., Makarevitch, I., Blinov, A. and Martin, J. 2001. Phylogeny of the genus Chironomus (Diptera) inferred from DNA sequences of mitochondrial Cytochrome b and Cytochrome oxidase I. - Molecular Phylogenetics and Evolution 19: 9-21.

Kaiser, T.S., Neumann, D., Heckel, D.G. and Berendonk, T.U. 2010. Strong genetic differentiation and postglacial origin of populations in the marine midge Clunio marinus (Chironomidae, Diptera). - Molecular Ecology 19: 2845-2857.

Krosch, M.N. 2011. Phylogeography of Echinocladius martini Cranston (Diptera: Chironomidae) in closed forest streams of eastern Australia. - Australian Journal of Entomology 50: 258-268.

Krosch, M.N. Baker, A.M. McKie, B.G. Mather, P.B. and Cranston P.S. 2009. Deeply divergent mitochondrial lineages reveal patterns of local endemism in chironomids of the Australian Wet Tropics. - Austral Ecology 34: 317-328.

Krosch, M.N., Baker, A.M., Mather, P.B., and Cranston, P.S. 2011a. Spatial population genetic structure reveals strong natal site fidelity in Echinocladius martini (Diptera: Chironomidae) in northeast Queensland, Australia. - Freshwater Biology 56: 1328-1341.

Krosch M.N., Baker A.M., Mather P.B. and Cranston, P.S. 2011b. Systematics and biogeography of the Gondwanan Orthocladiinae (Diptera: Chironomidae). - Molecular Phylogenetics and Evolution 59: 458-468.

Martin J., Guryev V., Blinov A. and Edward D.H.D. 2002. A molecular assessment of the extent of variation and dispersal between Australian populations of the genus Archaeochlus Brundin (Diptera: Chironomidae). - Invertebrate Systematics 16: 599-603.

Miller S.A., Dykes D.D., and Polesky H.F. 1988. A simple salting out procedure for extracting DNA from nucleated cells. - Nucleic Acids Research 16: 1215.

Pfenninger, M., Nowak, C., Kley, C., Steinke, D. and Streit, B. 2007. Utility of DNA taxonomy and barcoding for the inference of larval community structure in morphologically cryptic Chironomus (Diptera) species. - Molecular Ecology 16: 1957-1968. 
Sinclair C.S. and Gresens S. E. 2008. Discrimination of Cricotopus species (Diptera: Chironomidae) by DNA barcoding. - Bulletin of Entomological Research 98: 555-563.

Wang, Q., Wang, X. 2012. Comparison of methods for DNA extraction from a single chironomid for PCR analysis. - Pakistan Journal of Zoology 44: 421-426.

Webb J.M., Jacobus L.M., Funk D.H., Zhou X., Kondratieff B., Geraci, C.J., DeWalt, R.E., Baird, D.J., Richard, B., Phillips, I. and Hebert, P.D.N. 2012. A DNA Barcode Library for North American Ephemeroptera: Progress and Prospects. - PLOS ONE 7: e38063.

Wiederholm, T. 1983. Chironomidae of the Holarctic region. Keys and diagnoses. Part 1. Larvae. - Entomologica scandinavica, Supplement 19: 1-457.
Wiederholm, T. 1986. Chironomidae of the Holarctic region. Keys and diagnoses. Part 2. Pupae. - Entomologica scandinavica, Supplement 28: $1-482$

Willassen, E. 1999. "Take $25 \mathrm{mg}$ of insect.." - simmering signals of phylogeny from chironomid mitochondria. - Chironomus Newsletter on Chironomidae Reseach 12: 5-10.

Willassen, E. 2005. New species of Diamesa (Diptera: Chironomidae) from Tibet: conspecific males and females associated with mitochondrial DNA. - Zootaxa 1049: 19-32. 